\title{
Extended Composite Right/Left-Handed Transmission Line and Dual-Band Reactance Transformation
}

\author{
Yuming Zhang ${ }^{1}$ and Barry Spielman ${ }^{2}$ \\ ${ }^{1}$ Kunshan Industrial Technology Research Institute, 4F Technology Building, 1666 South Weicheng Road, \\ Kunshan, Jiangsu 215347, China \\ ${ }^{2}$ Department of Electrical and Systems Engineering, Washington University in St. Louis, One Brookings Drive, \\ Box 1127, St. Louis, MO 63130, USA
}

Correspondence should be addressed to Yuming Zhang, yumingzhang@live.com

Received 6 July 2009; Accepted 31 October 2009

Academic Editor: Hakan Kuntman

Copyright ( $) 2010$ Y. Zhang and B. Spielman. This is an open access article distributed under the Creative Commons Attribution License, which permits unrestricted use, distribution, and reproduction in any medium, provided the original work is properly cited.

\begin{abstract}
An extended composite right/left-handed transmission line is introduced, and its dual-band bandpass filter characteristics are explored. Novel reactance transformations, derived from this transmission line, are formulated to transform a low-pass prototype filter into a dual-band bandpass filter with arbitrary dual pass bands, well-defined in-band attenuation ripples, and high out-of-band rejection. The physical insight into such a dual-band bandpass filter is provided with a dispersion analysis. The transformations are verified by simulated results for dual-band bandpass filters.
\end{abstract}

\section{Introduction}

An extended composite right/left-handed (CRLH) transmission line is first introduced. Then, its fundamental properties are investigated. Consequently, a reactance transformation is derived for dual-band filter synthesis.

Although a dual-band bandpass filter design using the reactance transformation stems from the extended CRLH transmission line concept, it is totally different from the approach used by Tseng and Itoh [1]. In their approach, a traditional bandpass filter is first designed using quarterwave short circuited; then the dual-band bandpass filter with arbitrary two pass bands is implemented by replacing the microstrip lines with the CRLH transmission lines; the separation of these two pass bands is determined by the nonlinear phase slope of the CRLH tansmission lines. In our approach, a filter with arbitrary dual pass bands is directly transformed from a low-pass filter prototype; then the dual-band bandpass filter can be realized using traditional or metamaterial transmission lines; the separation of the two pass bands is precisely controlled by the reactance transformation.
In addition, Guan et al. reported dual-band bandpass filters employing two successive frequency transformations [2]. In their approach, the dual-band bandpass filter is also similar to the extended CRLH transmission line. The center frequencies of two pass bands are used to define frequency transformations; that is, the two pass bands are positioned by the center frequencies and their boundaries cannot be accurately specified. Additionally, their frequency transformations can only be used in a narrow band filter. However, our reactance transformation is parameterized by edge frequencies, and the bands are precisely delimited by them. Also, our reactance transformation can be used in an arbitrary band filter.

\section{Extended CRLH Transmission Line}

Figure 1 shows the equivalent circuit model for an extended CRLH transmission line. It consists of the series resonator comprised of $L_{1}$ and $C_{1}$ (Resonator 1), the series shunt resonator comprised of $L_{3}$ and $C_{3}$ (Resonator 3), the shunt shunt resonator comprised of $L_{2}$ and $C_{2}$ (Resonator 2), and the shunt series resonator comprised of $L_{4}$ and $C_{4}$ (Resonator 


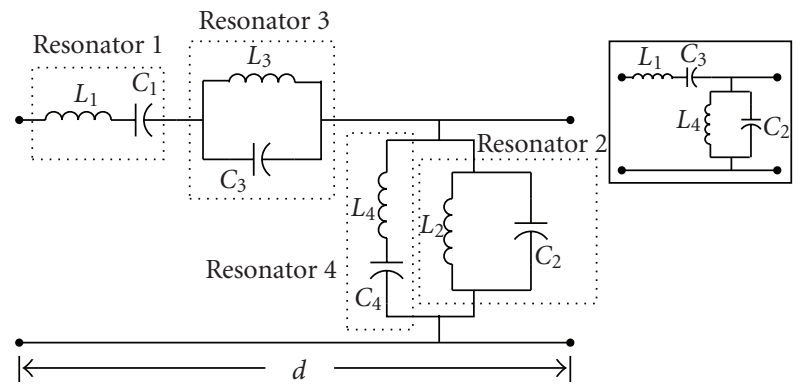

FIgure 1: A lumped-element model unit cell of an extended CRLH transmission line. $\left\{d\right.$ is the length of the unit cell. $L_{1}, L_{2}, L_{3}$, and $L_{4}$ are the inductances in henries/m. $C_{1}, C_{2}, C_{3}$, and $C_{4}$ are the capacitances in farads $/ \mathrm{m}$.

4). The elimination of Resonators 3 and 4 reduces the extended CRLH transmission line into a CRLH transmission line [3]. The extended CRLH transmission line becomes a dual CRLH transmission line [4] without Resonators 1 and 2 .

This paper focuses only on an extended CRLH transmission line that satisfies two conditions. The first condition is that the four resonators in Figure 1 have the same resonant frequency

$$
\omega_{0}^{2}=\frac{1}{L_{1} C_{1}}=\frac{1}{L_{3} C_{3}}=\frac{1}{L_{2} C_{2}}=\frac{1}{L_{4} C_{4}},
$$

where $\omega_{0}$ is the "center frequency." The series impedance $Z$ and shunt admittance $Y$ in Figure 1 are

$$
\begin{aligned}
& Z=j \omega L_{1}+\frac{1}{j \omega C_{1}}+\frac{1}{j \omega C_{3}+1 / j \omega L_{3}}, \\
& Y=j \omega C_{2}+\frac{1}{j \omega L_{2}}+\frac{1}{j \omega L_{4}+1 / j \omega C_{4}} .
\end{aligned}
$$

The second condition requires that the resonant frequencies of $Z$ and $Y$ coincide at $\omega_{0}^{\prime}$ :

$$
\left(\omega_{0}^{\prime}\right)^{2}=\frac{1}{L_{1} C_{3}}=\frac{1}{L_{4} C_{2}} .
$$

If either of the two conditions is not satisfied, three or more pass bands can occur.

2.1. Auxiliary CRLH Transmission Line. Here, an auxiliary CRLH transmission line is introduced to simplify the analysis of the extended CRLH transmission line. The auxiliary CRLH transmission line is comprised of the elements $L_{1}$, $C_{3}, L_{4}$, and $C_{2}$, as shown in the inset of Figure 1. From (4), it is known that the series and shunt resonant frequencies coincide at $\omega_{0}^{\prime}$. The high-pass cutoff frequency $\omega_{\text {UPPER }}^{\prime}$ and low-pass cutoff frequency $\omega_{\text {LOWER }}^{\prime}$ are expressed as follows [5]:

$$
\begin{aligned}
& \left(\omega_{\mathrm{UPPER}}^{\prime}\right)^{2} \\
& =\frac{1}{2}\left\{2\left(\omega_{0}^{\prime}\right)^{2}+\left(\omega_{R}^{\prime}\right)^{2}+\sqrt{\left[2\left(\omega_{0}^{\prime}\right)^{2}+\left(\omega_{R}^{\prime}\right)^{2}\right]^{2}-4\left(\omega_{0}^{\prime}\right)^{4}}\right\} \\
& =\frac{2}{2 /\left(\omega_{0}^{\prime}\right)^{2}+1 /\left(\omega_{L}^{\prime}\right)^{2}-\sqrt{\left[2 /\left(\omega_{0}^{\prime}\right)^{2}+1 /\left(\omega_{L}^{\prime}\right)^{2}\right]^{2}-4 /\left(\omega_{0}^{\prime}\right)^{4}}}, \\
& \left(\omega_{\mathrm{LOWER}}^{\prime}\right)^{2} \\
& =\frac{1}{2}\left\{2\left(\omega_{0}^{\prime}\right)^{2}+\left(\omega_{R}^{\prime}\right)^{2}+\sqrt{\left[2\left(\omega_{0}^{\prime}\right)^{2}+\left(\omega_{R}^{\prime}\right)^{2}\right]^{2}-4\left(\omega_{0}^{\prime}\right)^{4}}\right\} \\
& =\frac{2}{2 /\left(\omega_{0}^{\prime}\right)^{2}+1 /\left(\omega_{L}^{\prime}\right)^{2}+\sqrt{\left[2 /\left(\omega_{0}^{\prime}\right)^{2}+1 /\left(\omega_{L}^{\prime}\right)^{2}\right]^{2}-4 /\left(\omega_{0}^{\prime}\right)^{4}}},
\end{aligned}
$$

where $\left(\omega_{R}^{\prime}\right)^{2}=4 / L_{1} C_{2}$ and $\left(\omega_{L}^{\prime}\right)^{2}=1 / 4 L_{4} C_{3}$. From (5), three frequency relationships are obtained as follows:

$$
\begin{gathered}
\omega_{R}^{\prime}=\omega_{\mathrm{UPPER}}^{\prime}-\omega_{\mathrm{LOWER}}^{\prime}, \\
\frac{1}{\omega_{L}^{\prime}}=\frac{1}{\omega_{\mathrm{LOWER}}^{\prime}}-\frac{1}{\omega_{\mathrm{UPPER}}^{\prime}}, \\
\omega_{\mathrm{UPPER}}^{\prime} \omega_{\mathrm{LOWER}}^{\prime}=\omega_{L}^{\prime} \omega_{R}^{\prime}=\left(\omega_{0}^{\prime}\right)^{2} .
\end{gathered}
$$

2.2. Dispersion of the Extended CRLH Transmission Line. The dispersion analysis for the extended CRLH transmission line is presented here. Using the $A B C D$ matrix of the twoport network described in Figure 1 and the Bloch-Floquet theorem, the propagation constant $\gamma$ is determined by

$$
\begin{gathered}
\gamma=\alpha=\frac{1}{d} \arccos \left(\frac{Z Y+2}{2}\right), \quad \text { if }-4<Z Y<0, \\
\gamma=j \beta=\frac{j}{d} \operatorname{arccosh}\left(\frac{Z Y+2}{2}\right), \quad \text { if } Z Y<-4 \text { or } Z Y>0,
\end{gathered}
$$

where $Z$ and $Y$ are defined in (2) and (3), respectively. Equation (9) corresponds to an evanescent wave whereas (10) represents a propagating wave. $Z Y$ can be written as the follows:

$$
Z Y=-4\left(\frac{w}{\omega_{R}^{\prime}}-\frac{\omega_{L}^{\prime}}{w}\right)^{2}
$$

where

$$
w=\omega_{0}\left(\frac{\omega}{\omega_{0}}-\frac{\omega_{0}}{\omega}\right) .
$$

$\omega_{R}^{\prime}$ and $\omega_{L}^{\prime}$ are defined in (6) and (7), respectively. $Z Y=0$ gives two resonant frequencies:

$$
\begin{aligned}
& \omega_{\text {ResUpper }}=\frac{1}{2}\left[\sqrt{4 \omega_{0}^{2}+\left(\omega_{0}^{\prime}\right)^{2}}+\omega_{0}^{\prime}\right], \\
& \omega_{\text {ResLower }}=\frac{1}{2}\left[\sqrt{4 \omega_{0}^{2}+\left(\omega_{0}^{\prime}\right)^{2}}-\omega_{0}^{\prime}\right] .
\end{aligned}
$$


$Z Y=-4$ yields four cutoff frequencies:

$$
\begin{aligned}
& \omega_{U U}=\frac{1}{2}\left[\sqrt{4 \omega_{0}^{4}+\left(\omega_{\mathrm{UPPER}}^{\prime}\right)^{2}}+\omega_{\mathrm{UPPER}}^{\prime}\right], \\
& \omega_{U L}=\frac{1}{2}\left[\sqrt{4 \omega_{0}^{4}+\left(\omega_{\mathrm{LOWER}}^{\prime}\right)^{2}}+\omega_{\mathrm{LOWER}}^{\prime}\right], \\
& \omega_{L U}=\frac{1}{2}\left[\sqrt{4 \omega_{0}^{4}+\left(\omega_{\mathrm{LOWER}}^{\prime}\right)^{2}}-\omega_{\mathrm{LOWER}}^{\prime}\right], \\
& \omega_{L L}=\frac{1}{2}\left[\sqrt{4 \omega_{0}^{4}+\left(\omega_{\mathrm{UPPER}}^{\prime}\right)^{2}}-\omega_{\mathrm{UPPER}}^{\prime}\right] .
\end{aligned}
$$

These frequencies are related as follows:

$$
\omega_{0}^{2}=\omega_{\text {ResUpper }} \omega_{\text {ResLower }}=\omega_{U U} \omega_{L L}=\omega_{U L} \omega_{L U} .
$$

Equations (5) and (8) can be used to show that $\omega_{\text {UPPER }}^{\prime}>$

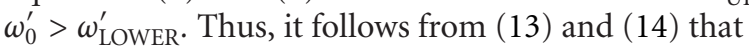

$$
\omega_{U U}>\omega_{\text {ResUpper }}>\omega_{U L}>\omega_{0}>\omega_{L U}>\omega_{\text {ResLower }}>\omega_{L L} .
$$

Based upon (9) and (10), the dispersion of the extended CRLH transmission line is plotted in Figure 2(a). It is noted that the four cutoff frequencies define key features of the dual passband behavior. $\omega_{L L}$ and $\omega_{L U}$ are the lower and upper edges of the lower pass band, respectively. $\omega_{U L}$ and $\omega_{U U}$ are the lower and upper edges of the upper pass band, respectively. A wave exhibits phase-backward propagation when the operating frequency is between $\omega_{L L}$ and $\omega_{\text {ResLower, }}$ or between $\omega_{U L}$ and $\omega_{\text {ResUpper. }}$. However, a wave exhibits phase-forward propagation when the operating frequency is between $\omega_{\text {ResLower }}$ and $\omega_{L U}$, or between $\omega_{\text {ResUpper }}$ and $\omega_{U U}$. Otherwise, the wave is evanescent. It is noteworthy that $\omega_{\text {ResLower }}$ and $\omega_{\text {ResUpper }}$ are transition points between forward and backward waves. The performance between $\omega_{\text {ResLower }}$ and $\omega_{\text {ResUpper }}$ is similar to that of a dual CRLH transmission line [4] whereas the performance below $\omega_{\text {ResLower }}$ or greater than $\omega_{\text {ResUpper }}$ is similar to that of a CRLH transmission line $[3,5]$.

Figure 2(b) represents the equivalent circuit behavior of the extended CRLH transmission line at different frequencies. When the operating frequency is less than $\omega_{\text {ResLower, }}$ the extended CRLH transmission line is equivalent to a pure left-handed transmission line. Thus, the high-pass cutoff frequency $\omega_{L L}$ appears. When the operating frequency is between $\omega_{\text {ResLower }}$ and $\omega_{0}$, the extended CRLH transmission line is equivalent to a pure right-handed transmission line with the low-pass cutoff frequency $\omega_{L U}$. When the operating frequency is between $\omega_{0}$ and $\omega_{\text {ResUpper }}$, the extended CRLH transmission line is equivalent to a pure left-handed transmission line with the high-pass cutoff frequency $\omega_{U L}$. When the operating frequency is greater than $\omega_{\text {ResUpper, }}$, the extended CRLH transmission line is equivalent to a pure right-handed transmission line with the low-pass cutoff frequency $\omega_{U U}$.
2.3. Important Frequency Expressions. Using (6), (7), and (14), $\omega_{R}^{\prime}$ and $\omega_{L}^{\prime}$ are rewritten by

$$
\begin{aligned}
& \omega_{R}^{\prime}=\left(\omega_{U U}-\omega_{L L}\right)-\left(\omega_{U L}-\omega_{L U}\right), \\
& \frac{1}{\omega_{L}^{\prime}}=\frac{1}{\left(\omega_{U L}-\omega_{L U}\right)}-\frac{1}{\left(\omega_{U U}-\omega_{L L}\right)} .
\end{aligned}
$$

Equations (17), (18), and (15) are important expressions that are used in Section 3 to transform frequency and elements.

It should be noted that all formulations presented in Section 2 are derived from the infinite extended CRLH transmission line. In practice, no transmission line is infinite. However, it has been verified in [3] that the infinitestructure approximation provides reasonable accuracy when a sufficiently large number $(>3)$ cells are used.

\section{Dual-Band Reactance Transformation}

In this section, the dual-band frequency and element transformations are derived. The frequency transformation is a reactance function and converts a low-pass prototype filter with well-defined insertion loss [6] to a dual-band bandpass filter. Simulation results are presented to verify the transformations.

3.1. Dual-Band Bandpass Frequency Transformations. The desired frequency transformation is obtained by replacing the reactance and the susceptance in the prototype filter by series and shunt resonators such that

$$
\begin{aligned}
& j \Omega L=Z, \\
& j \Omega C=Y,
\end{aligned}
$$

where $\Omega, L$ and $C$ are the normalized frequency, inductance, and capacitance of the low-pass prototype filter. $Z$ and $Y$ are defined in (2) and (3), respectively. Multiplying the right and left sides of (19) and (20), respectively, the dual-band bandpass frequency transformation is obtained as follows:

$$
\Omega^{2}=\left(\frac{w}{\omega_{R}^{\prime}}-\frac{\omega_{L}^{\prime}}{w}\right)^{2},
$$

where the definitions of $w, \omega_{R}^{\prime}$ and $\omega_{L}^{\prime}$ are given in (12), (17) and (18), respectively. The normalized low-pass cutoff frequency, $\Omega_{R}^{2}=4 / L C=1$, of the pure right-handed transmission line is used for the derivation of (21). With the substitution of $w$, the explicit expression of $w / \omega_{R}^{\prime}$ shows it is a low-pass to bandpass transformation with the passband bandwidth of $\omega_{R}^{\prime}[6] . \omega_{L}^{\prime} / w$ is a low-pass to bandstop transformation with the stopband bandwidth of $\omega_{L}^{\prime}[6]$. The frequency mappings of (21) are depicted in Figure 3. It is observed that the subtraction of the low-pass to bandstop mapping from the low-pass to bandpass mapping gives the low-pass to dual-band bandpass mapping. 


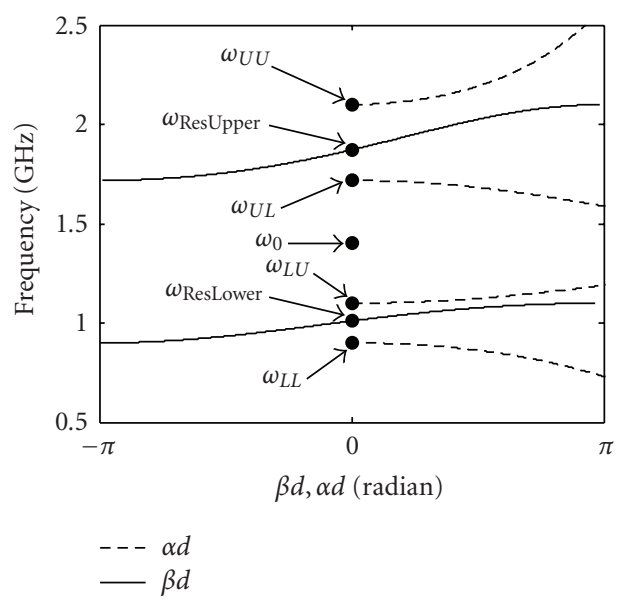

(a)

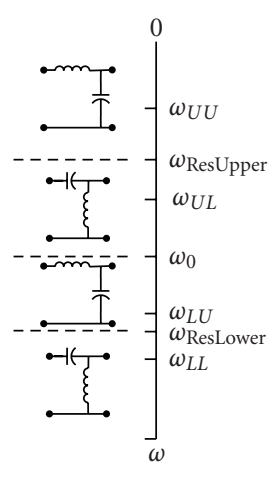

(b)

Figure 2: The dispersion (a) and equivalent circuits (b) of the extended CRLH transmission line ( $\beta$ and $\alpha$ are defined in (9) and (10).)

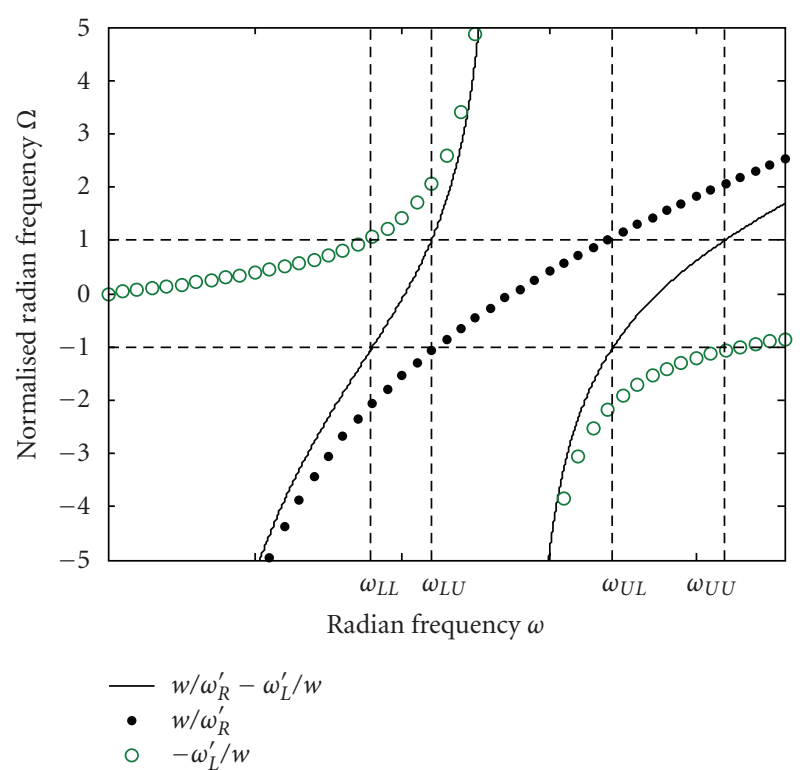

FIGURE 3: Mapping from normalized frequency of a prototype lowpass filter into actual filter frequency. (The solid line, plotted by $\Omega=$ $w / \omega_{R}^{\prime}-\omega_{L}^{\prime} / w$, is for the dual-band bandpass filter. The circle and dot lines describe the bandstop transformation with minus sign, $\Omega=$ $-\omega_{L}^{\prime} / w$, and the bandpass transformation, $\Omega=w / \omega_{R}^{\prime}$, resp. Here, $w=\omega_{0}\left(\omega / \omega_{0}-\omega_{0} / \omega\right)$.)

3.2. The Element Transformations. Substituting $\Omega$ with (21) in (19) and (20), and using (1), the element transformations are obtained as follows:

$$
\begin{array}{ccc}
L_{1}=\frac{L}{\omega_{R}^{\prime}}, & C_{1}=\frac{1}{L_{1} \omega_{0}^{2}}, \quad C_{2}=\frac{C}{\omega_{R}^{\prime}}, \quad L_{2}=\frac{1}{C_{2} \omega_{0}^{2}}, \\
C_{3}=\frac{1}{L \omega_{L}^{\prime}}, & L_{3}=\frac{1}{C_{3} \omega_{0}^{2}}, \quad L_{4}=\frac{1}{C \omega_{L}^{\prime}}, \quad C_{4}=\frac{1}{L_{4} \omega_{0}^{2}} .
\end{array}
$$
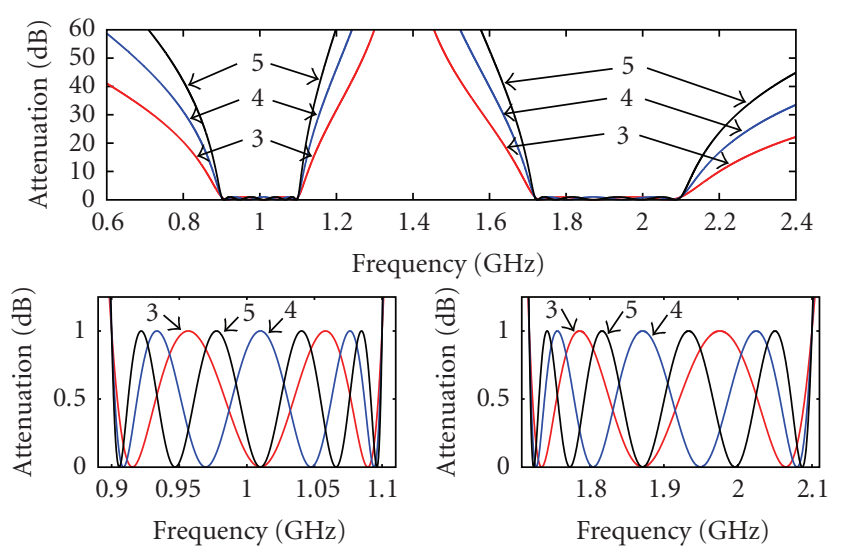

Figure 4: Simulated attenuation responses of the dual-band bandpass filters transformed from 1-dB Chebyshev low-pass filters for filter orders of 3, 4, and 5. (The response of the lower and upper pass bands in the upper subplot is amplified in the left and right bottom subplots, resp.)

It is observed that $L_{1}, C_{1}, L_{2}$, and $C_{2}$ constitute a bandpass filter with the center frequency $\omega_{0}$ and passband bandwidth of $\omega_{R}^{\prime} . L_{3}, C_{3}, L_{4}$, and $C_{4}$ form a band stop filter with center frequency $\omega_{0}$ and a stop band bandwidth of $\omega_{L}^{\prime}$.

3.3. Simulations. Dual-band bandpass filter, specified by the band edge frequencies of $0.9 \mathrm{GHz}, 1.1 \mathrm{GHz}, 1.718 \mathrm{GHz}$, and $2.1 \mathrm{GHz}$, was transformed from a Chebyshev low-pass filter with $1-\mathrm{dB}$ passband ripple using (22). Figure 4 shows simulated attenuation responses of lumped-element dualband bandpass filters of orders 3, 4, and 5. The lower and upper pass bands have the same ripple magnitude. The attenuation tends to infinity at the center frequency $\omega_{0}=1.375 \mathrm{GHz}$. The observed pass band center frequencies, $1.01 \mathrm{GHz}$ and $1.871 \mathrm{GHz}$, agree with the computed $\omega_{\text {ResLower }}$ and $\omega_{\text {ResUpper }}$, respectively. 


\section{Conclusion}

The extended CRLH transmission line has been introduced and characterized. Novel dual-band bandpass reactance transformations were derived. A dual structure of the extended CRLH transmission line can be explored using an approach similar to that described here. A dual-band bandstop frequency transformation can be obtained by replacing $\Omega$ by $-1 / \Omega$.

\section{References}

[1] C.-H. Tseng and T. Itoh, "Dual-band bandpass and bandstop filters using composite right/left-handed metamaterial transmission lines," in Proceedings of the IEEE MTT-S International Microwave Symposium Digest, pp. 931-934, San Francisco, Calif, USA, June 2006.

[2] X. H. Guan, Z. Ma, P. Cai, et al., "A dual-band bandpass filter synthesized by using frequency transformation and circuit conversion technique," in Proceedings of the IEEE Asia-Pacific Microwave Conference (APMC '05), vol. 4, Yokohama, Japan, December 2005.

[3] C. Caloz and T. Itoh, Electromagnetic Metamaterial; Transmission Line Theory and Microwave Applications: The Engineering Approach, John Wiley \& Sons, Hoboken, NJ, USA, 2006.

[4] C. Caloz, "Dual composite right/left-handed (D-CRLH) transmission line metamaterial," IEEE Microwave and Wireless Components Letters, vol. 16, no. 11, pp. 585-587, 2006.

[5] Y. Zhang and B. E. Spielman, "A stability analysis for timedomain method-of-moments analysis of 1-D double-negative transmission lines," IEEE Transactions on Microwave Theory and Techniques, vol. 55, no. 9, pp. 1887-1898, 2007.

[6] G. L. Matthaei, L. Young, and E. M. T. Jones, Microwave Filter, Impedance-Matching Networks, and Coupling Structures, Artech House, Dedham, Mass, USA, 1980. 

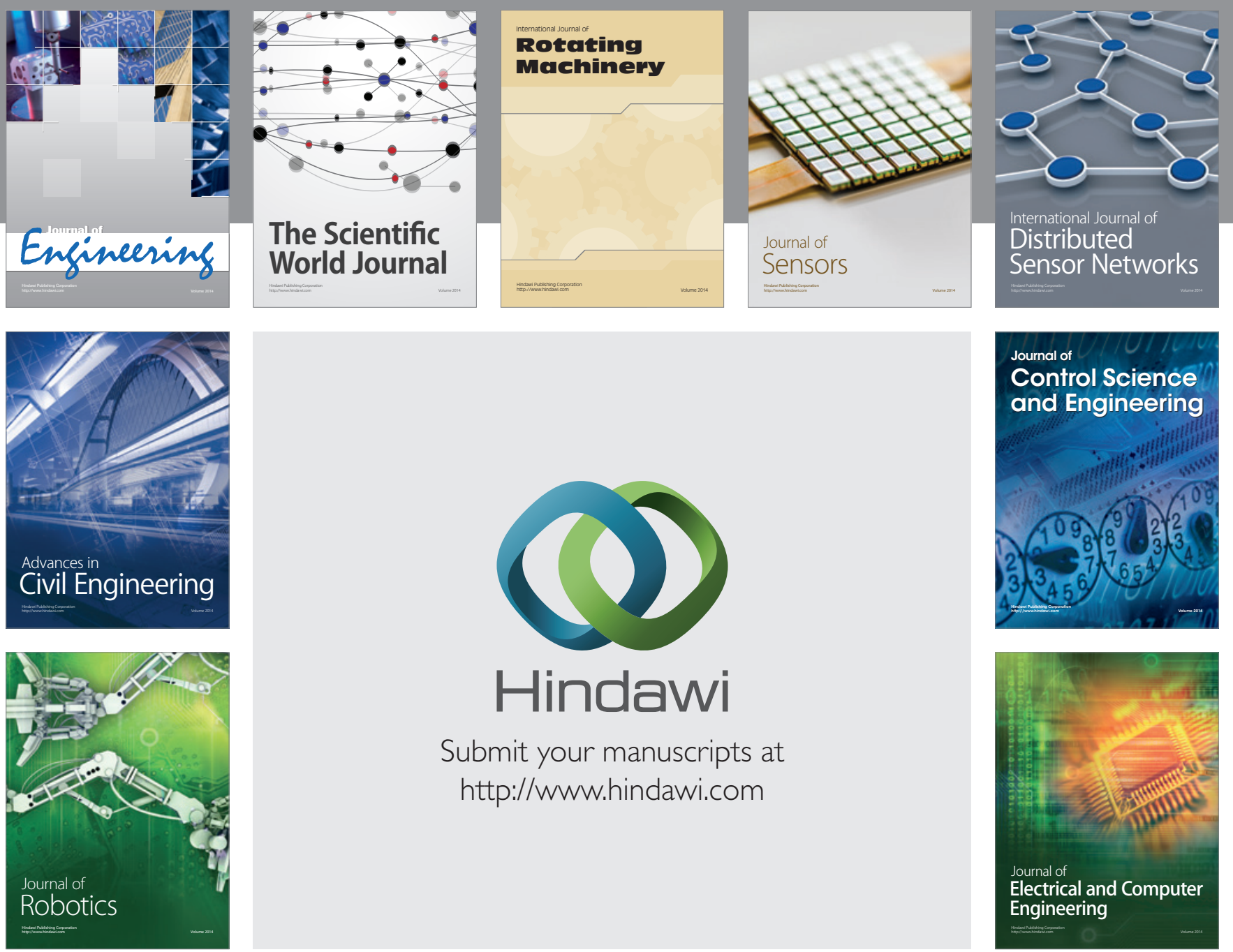

Submit your manuscripts at

http://www.hindawi.com
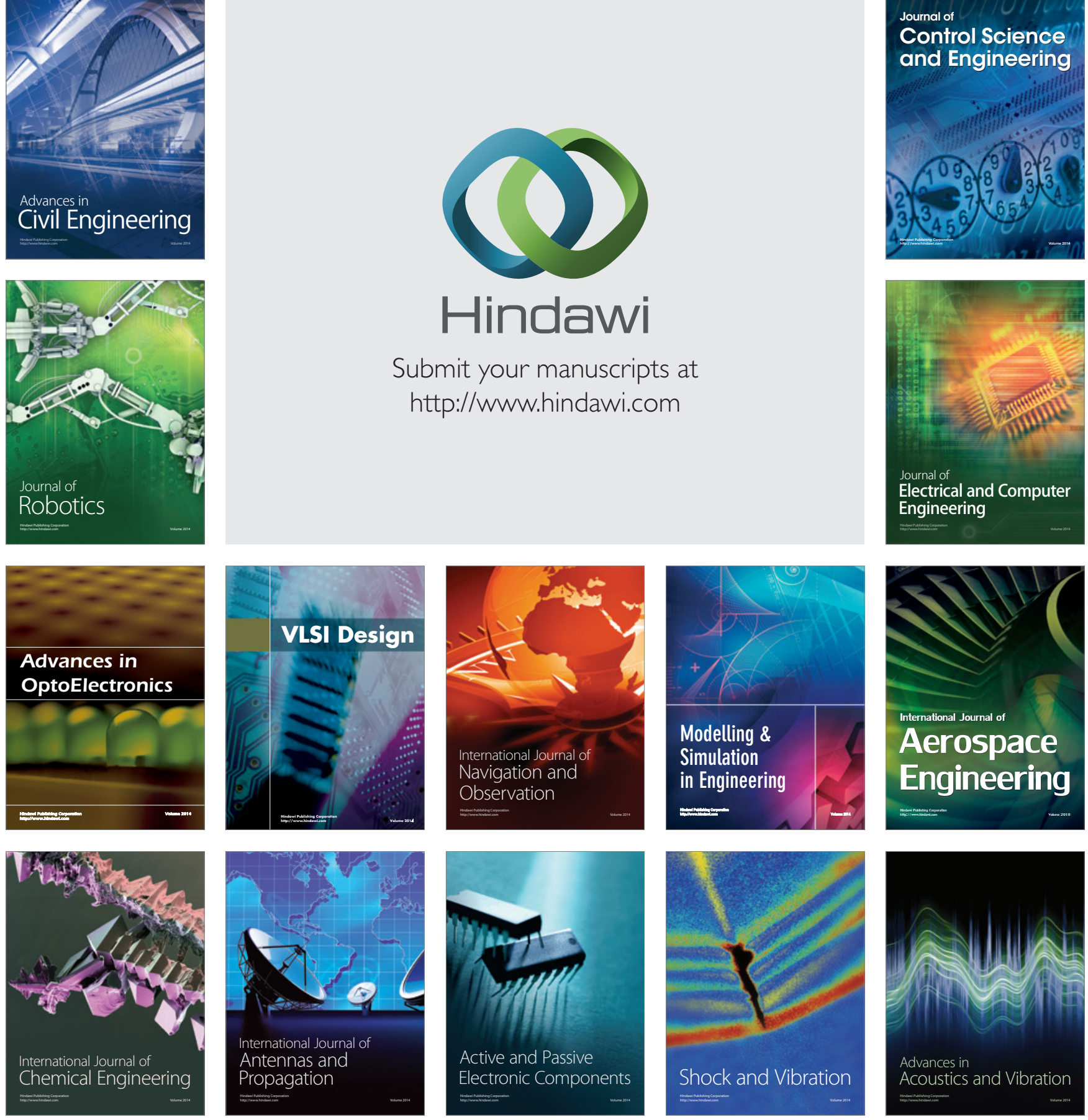